\title{
Investigating decision-making in Parkinson's disease in tasks characterized by probabilistic cue-outcome associations: the role of optimality
}

\author{
Agata Ryterska' ${ }^{1}$, Adam J. L. Harris ${ }^{2}$, Anastasia Ejova ${ }^{3}$, Marjan Jahanshahi ${ }^{4}$ and Magda Osman ${ }^{*}$
}

*Correspondence: m.osman@qmul.ac.uk

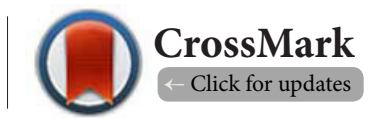

'Biological and Experimental Psychology, School of Biological and Chemical Sciences, Queen Mary University of London, Mile End Road, London, E1 4NS, UK.

${ }^{2}$ Department of Cognitive, Perceptual and Brain Sciences, Division of Psychology and Language Sciences, University College London, 26 Bedford Way, London, WC1H OAP, UK.

${ }^{3}$ School of Psychology, The University of Adelaide, Adelaide, SA 5005, Australia.

${ }^{4}$ Cognitive Motor Neuroscience Group, Sobell Department of Motor Neuroscience and Movement Disorders, UCL Institute of Neurology, 33 Queen Square, London, WC1N 3BG, UK.

\begin{abstract}
Is probabilistic information one of the critical factors contributing to decision-making impairments in Parkinson's disease (PD)? The results of many studies may suggest that in fact it is, and that PD patients struggle to process probabilities accurately. However, a growing body of evidence suggests otherwise. To explore this issue further, a novel gambling scenario was developed in order to compare decision-making behaviour in the form of betting in three different set ups: deterministic, probabilistic, and unpredictable. Participants' behaviour was analysed using newly developed metrics, designed to examine optimal betting strategies. In our novel task PD patients performed as well as age-matched healthy controls (OHCs) across different set ups. Thus, in answer to the question posed, probabilistic information persedoes not seem to be the critical factor in causing impaired decision-making in PD.
\end{abstract}

Keywords: Parkinson's disease, decision-making, optimality, gambling, probabilistic environment

\section{Introduction}

Parkinson's disease (PD) is a neurodegenerative disorder primarily affecting dopamine producing cells in substantia nigra pars compacta. In addition to motor symptoms, such as akinesia, bradykinesia, tremor and rigidity, PD is known to affect executive functions [1-3] including decision-making, which is the focus of the present study. Much of the evidence showing impaired decision-making in patients with PD comes from probabilistic decision-making tasks [4-11].

An example of a probabilistic decision-making task on which patients with PD have typically been found to be impaired is the Weather Prediction Task (WPT). In this task participants need to predict which weather outcome (i.e., 'sun' or 'rain') is more likely to occur, based on a combination of 4 cards presented to them. Each card is associated with a different probability of each outcome occurring. Participants need to learn about the structure of the task (i.e., the probabilities associated with different cards) by utilizing discreet feedback provided after each trial (i.e., correct vs. incorrect) to make decisions regarding the most likely weather outcome. When compared to healthy age matched controls (OHCs) PD patients on medication show impaired performance on this task $[\mathbf{7 , 8}, \mathbf{1 0}, \mathbf{1 2}-15]$, which was taken as evidence that they have problems in learning the probabilistic structure of the environment $[8,16]$ which in turn affects their decisions.

In WPT and other tasks of this kind used to examine decisionmaking in PD (e.g., Iowa Gambling Task [IGT], Cambridge Gambling Task [CGT]) people need to learn about the reliability and validity of information that predicts an outcome in order to improve the accuracy of their decisions over the course of the task. To achieve this, theyoften focus on outcome information associated with their choices-such as rewards and feedback $[17,18]$. Crucially, these decision-making tasks (e.g., WPT-18; IGT-17) are constructed in such a way that the relationship 
between the cue and outcome is probabilistic (e.g., rain [outcome] will occur with probability 0.8 whenever a cloud [cue] is present). Because of the converging evidence showing that patients with $\mathrm{PD}$ are impaired on these decision-making tasks characterized by a probabilistic relationship between cues and outcomes, it was hypothesized that processing of probabilistic cue-outcome information is impaired in $\mathrm{PD}[\mathbf{8}, \mathbf{1 6 , 1 9 , 2 0}]$.

This impairment has been attributed to dopamine depletion associated with neurological changes characteristic of PD $[6,8,9,15,21-24]$ or, alternatively, to the dopaminergic medication administered as part of PD management $[\mathbf{4 , 2 5 - 3 0}]$. The latter hypothesis was associated with the observation that PD patients seem to perform just as well as OHCs on certain decisionmaking tasks (such as most gambling tasks) when tested OFF dopaminergic medication $[4,7,26,28,31-36]$ while being impaired when tested ON their usual medication. The reason why increasing dopamine levels through medication adversely affects decision-making has been explained by the dopamine 'overdosing' hypothesis $[\mathbf{7 , 2 6 , 3 0 , 3 6 , 3 7 ]}$. This hypothesis states that dopaminergic medication improves cognitive functions reliant on brain areas which have depleted levels of dopamine (e.g., putamen and dorsal caudate). However, L-dopa also increases levels of dopamine in less affected brain areas (e.g., ventral striatum), which leads to overdosing and as a consequence to dysfunction of other cognitive processes such as detecting probabilistic cue-outcome associations $[\mathbf{2 6 , 3 0 ]}$.

The problem with stating that PD patients are impaired when it comes to detecting probabilistic cue-outcome associations is that there is conflicting evidence which complicates matters. For example, PD patients have been shown to perform just as well as healthy controls on very complex probabilistic tasks, such as the Dynamic Decision Making (DDM) task [38-40]. In DDM tasks, participants are presented with a complex, dynamic environment in which the association between cues and outcomes is probabilistic. They are required to manipulate the cues present to obtain a desirable outcome, but here they receive cumulative feedback after each choice they make. The consensus is that studies utilizing tasks of this kind tend not to find impairments to performance in PD patients because the feedback structure facilitates learning [38-40]. This evidence shows that under certain circumstances (e.g., when there is no feedback or feedback is cumulative) PD patients can perform just as well as OHCs in probabilistic tasks $[\mathbf{3 8 , 3 9 , 4 1 - 4 3 ]}$, thus suggesting that factors other than the probabilistic element of the task may in fact be responsible for decision-making impairments observed in PD.

How might these conflicting results be settled? To uncover the extent to which a probabilistic cue-outcome relationship is the critical factor in inducing impaired decision-making in patients with $\mathrm{PD}$, one approach would be to compare the same probabilistic task under different feedback conditions. However, this approach still has limitations as to the extent to which probabilistic cue-outcome relations are the root cause of impairments to decision-making in PD patients, as it does not inform us about how performance in probabilistic condition compares to performance when cue-outcome relations are of a different type. An alternative approach is to compare decisionmaking performance in a probabilistic version of a task with performance on deterministic (e.g., outcome B occurs with probability 1 when Cue A is present) and unpredictable (e.g., outcome $\mathrm{B}$ occurs with probability $p$ when Cue A is present, and the same probability, $p$, when Cue $A$ is absent) versions. If we have an idea of how good $\mathrm{PD}$ patients' performance is when presented with different cue-outcome relationships, when all other things are equal, we can establish whether impairments in decision-making are specifically associated with probabilistic cue-outcome situations.

To address this issue, an important factor that needs to be taken into account is what we mean by good performance on a decision-making task.Usually, good performanceon a decisionmaking task is defined in relation to performance levels achieved by young and older HCs (YHC and OHC respectively) as the benchmark. However, outside of the between-groups approach, if we look to the specific decision-making tasks, we also need to establish from the outset what is deemed as optimal performance given the specific structure of the cue-outcome relationship in a task [44]. To illustrate, take for example a hypothetical gambling scenario in which, on each round of play, people choose how much money to bet (low, medium, high) on a certain outcome occurring. If the outcome is perfectly predictable (i.e., deterministic) then people will have higher final winnings than if the outcome is only partially predictable or completely unpredictable, even if in all these cases the betting behavior is perfectly optimal. For example, the gambling scenario could be set up in such a way that in the deterministic condition favorable outcome occurs on every third trial (i.e., round of play), in the probabilistic condition the favorable outcome occurs on every third trial with a probability of $66 \%$ and on other trials with a probability of $17 \%$, and in the unpredictable condition the favorable outcome occurs on a third of all trials, but the distribution of these trials is randomly assigned. Crucially, the total number of favorable outcomes is similar in all three conditions, but the optimal strategy is different in the different conditions (see Table 1). In the deterministic and probabilistic conditions the strategy that should yield the highest returns is to bet low on the first two trials and high on every third trial. In the unpredictable condition the optimal strategy is to bet low all the time in order to minimise potential losses. These different strategies, even though optimal, are bound to result in different amounts of final wins (i.e., the total amount of points won in the task) if applied throughout the task.

To clarify this further, consider the following example. Let's imagine a gambling scenario in which participants are repeatedly facing a choice between three bets. The structure of potential wins is that whenever you bet 10 you can win 27 points, whenever you bet 20 you can win 54 points, and whenever you bet 30 you can win 81 points. The gain from each of these gambles is calculated as 'points you win' minus 'points you had 
Table 1. Summary of optimal strategies indeterministic, probabilistic, and unpredictable conditions.

\begin{tabular}{lll}
\hline Deterministic condition & Probabilistic condition & Unpredictable condition \\
\hline A win on every third shot & $33 \%$ average probability of winning; 66\% & $33 \%$ average probability of winning \\
Loss (L), Loss (L), Win (W) ... and so & probability of win on every third shot & Optimal strategy (to minimize losses): \\
on for 50 trials & $83 \%$ probability of L, 83\% probability of & Bet 10 always \\
Optimal strategy: Bet 10, Bet 10, Bet 30 & L, 66\% probability of W... and so on for & \\
& 50 trials \\
& Optimal strategy: Bet 10, Bet 10, Bet 30 & \\
\hline
\end{tabular}

to bet', so the gain from the bets described above would be 17 , 34 , and 51, respectively. Consequently, assuming that there are 50 trials (16 of which are winning trials), and that participants start with 2000 points, the highest return in the deterministic condition would be expected when participants bet low (i.e., 10) on all of the non-favorable outcome trials and high (i.e., 30) on all of the favorable outcome trials $\left[2000+\left(16^{\star} 51\right)-\left(34^{\star} 10\right)\right.$ $=2476$ ]. In the probabilistic scenario, if participants respond optimally (i.e., bet 10) on a non-favorbale outcome trial,they have $83 \%$ chance of losing 10 , but a $17 \%$ chance of winning 17. Therefore, the expected value $(\mathrm{EV})$ of this gamble is $(0.83$ $\mathrm{x}-10)+(0.17 \mathrm{x} 17)=-5.41$. On a favorable outcome trial, on the other hand, participants have $66.66666 \%$ chance of winning 51 , but a $33.333333 \%$ chance of losing 30, when betting optimally. Therefore, the EV is $(0.666666 \times 51)+(0.3333333 x-30)=24$. As there are 16 'winning' trials and 34 'losing' trials, the expected gain across the game is $(-5.41 \times 34)+(24 \times 16)=200.06$. Therefore, the expected final outcome with optimal responding is 2200.06 . Finally, in an unpredictable scenario all trials are associated with the same probability of winning, and on each one participants have a $66.666666 \%$ chance of losing 10 , and a $33.333333 \%$ chance of winning 17, assuming the optimal strategy. Therefore, $\mathrm{EV}$ on a trial is $(0.6666 \mathrm{x}-10)+(0.333333 \times 17)=-1$. Because there are 50 trials, the expected gain across the game is $(50 x-1)=-50$. Consequently, the expected final amount after 50 trials is 1950. If participants adopted any other strategy in the deterministic, probabilistic or unpredictable conditions than those described above, this would lower the value of expected final amount. Consequently, the strategies described above are the optimal strategies to adopt in each particular scenario, even though they differ in terms of how much one can expect to win when utilizing them.

Table 2 presents the scores that could be expected if participants utilised the optimal strategy in each condition. It is clear that the final scores expected in the deterministic condition are much higher than those expected in the probabilistic and unpredictable conditions. At the same time, the difference in the final score achieved does not mean that participants in the probabilistic and unpredictable conditions are employing a 'worse strategy' than participants in the deterministic condition. Judging participants' performance on the task by the final score they have achieved might, therefore, lead to the wrong conclusion in terms of how good participant performance actually is. Consequently, establishing how well participants can be expected to perform in different conditions, and comparing that optimal score with the actual score is crucial for the objective assessment of the participants' performance in different decision-making conditions, which will be a priority in our study.

Furthermore, as Harris and Osman [44] pointed out, in addition to differences between conditions based on employing the optimal strategy, there are also differences between conditions based on the cost of employing the worst strategy. For example, if in the probabilistic gambling scenario described above participants employed the worst possible strategy (i.e., betting high, high and then low repeatedly) their scores would be much lower than the scores of participants employing the worst possible strategy (i.e., betting high all the time) in the unpredictable condition, as illustrated by Table 2. Consequently, the difference between final scores and optimal scores in the task also needs to be considered in the context of how badly participants can actually do in different conditions. The important point concerning the discussion of optimal strategy and worstpossible strategy is that the basis on which the experimenter chooses to assess how good participants' behavior is in a given condition will affect the conclusions that can be drawn about their performance. Moreover, this will also impact on the level of impairments to decision-making that can be inferred in

Table 2. Final scores in the three different conditions (deterministic, probabilistic, unpredictable) depending on the strategy used ${ }^{*}$.

\begin{tabular}{llll}
\hline & \multicolumn{3}{c}{ Condition } \\
\hline & Deterministic & Probabilistic & Unpredictable \\
\hline $\begin{array}{l}\text { Optimal strategy } \\
\text { final score }\end{array}$ & 2476 & 2200 & 1950 \\
$\begin{array}{l}\text { Worst strategy } \\
\text { final score }\end{array}$ & 1252 & 1567 & 1850 \\
\hline
\end{tabular}

${ }^{*}$ Assuming that there were 50 trials, $1 / 3$ of which were winning trials. Betting options available were 10,20 , or 30 credits, and they were associated with potential wins of $27,54,81$.

clinical populations, in this case, patients with PD. Consequently, to assess the extent to which PD patients' decision-making is optimal (or sub-optimal) when the cue-outcome relationship is probabilistic, we not only need to compare decision-making in different conditions and in comparison to young and older $\mathrm{HCs}$, but also in relation to the optimal and worst possible 
performance in a particular condition.

Therefore, in this study we set out to assess decision-making in patients with PD in different types of conditions (deterministic, probabilistic, and unpredictable), while taking into consideration what optimal behavior means in these conditions. For that reason we utilized a task that allows direct comparison between probabilistic, deterministic and unpredictable conditions and in which optimal strategy can easily be defined. The task was designed to resemble a decision-making scenario participants would have knowledge of. Gambling scenarios are often used in studies of decision-making in PD patients to assess the extent of impairments (such as GDT, IGT) [11], which is why we also used this type of framing for our study.

\section{Methods}

To examine how different types of task conditions influence decision-making, performance on a gambling task in people with PD and OHCs was investigated. YHCs were also tested to provide an additional baseline level of performance for each task condition.

\section{Participants}

Forty four university students (YHCs), 25 PD patients and 26 OHCs participated in the study. Demographic data of participants is presented in Table 3. Patients were recruited from the movement disorders clinic at the National Hospital for Neurology and Neurosurgery. All patients were non-demented, as demonstrated by scores above 24 on the Mini-Mental State Examination (MMSE) [45]. Screening for depression using the Beck Depression Inventory-II (BDI-II) [46] revealed 4 PD patients scored in the depressed range (score $>18$ ). However, none of the patients had a clinical diagnosis of depression, and none were on anti-depressants at the time of the study. Stage of illness was assessed using the Hoehn and Yahr scale [47] and disability with the Schwab and England Activities of Daily Living scale [48]. All patients where in the mild to moderate stages of the disease with scores on the Hoehn and Yahr scale ranging from 1 to $4(\mathrm{M}=1.98, \mathrm{SD}=0.90)$. On the Schwab and England scale scores ranged from 5 to $9(\mathrm{M}=7.84, \mathrm{SD}=1.02)$.

Table 3. Demographic characteristics of participants.

\begin{tabular}{|c|c|c|c|c|c|c|c|}
\hline & \multicolumn{6}{|c|}{ Group } & \multirow[b]{3}{*}{ p value } \\
\hline & \multicolumn{2}{|c|}{ PDs } & \multicolumn{2}{|c|}{ OHCs } & \multicolumn{2}{|c|}{ YHCs } & \\
\hline & Mean & SD & Mean & SD & Mean & SD & \\
\hline Age & 69.69 & 7.33 & 67 & 7.2 & 20.86 & 3.75 & -- \\
\hline Gender (M/F) & $16 / 9$ & -- & $6 / 20$ & -- & $11 / 33$ & -- & -- \\
\hline MMSE & 29.04 & 1.1 & 29.62 & 0.7 & -- & -- & 0.032 \\
\hline BDI & 10.44 & 6.82 & 5 & 3.66 & -- & -- & $<0.001$ \\
\hline $\begin{array}{l}\text { Schwab \& } \\
\text { England scale }\end{array}$ & 7.84 & 1.02 & -- & -- & -- & -- & -- \\
\hline $\begin{array}{l}\text { Hoehn \& } \\
\text { Yahr scale }\end{array}$ & 1.98 & 0.9 & -- & -- & -- & -- & -- \\
\hline
\end{tabular}

All patients were examined while on dopaminergic medication. Every participant from PD and OHC groups received money to cover their travel expenses or 10 pounds for taking part in the experiment.

\section{Design and materials}

The study used a $3 \times 3$ parametric design with between-subjects variables of Group (PD patients, OHCs, YHCs) and Task Condition (Deterministic, Probabilistic, and Unpredictable).

The visual layout of the screen, cover story, and main instructions were identical for the Deterministic, Probabilistic and Unpredictable conditions. Participants were told that they were going to see a football player (soccer player), Ronaldinho, taking free kicks. For each free kick they had to bet 10,20 or 30 credits on Ronaldinho scoring a goal. They were told that the aim of the task was to earn as many credits as possible by betting appropriately given the outcome. Each bet was associated with different amounts of credit that could be won $(27,54$, and 81 , respectively). Each condition consisted of 50 trials, $33 \%$ of which were winning ('goal') trials (i.e., the favorable outcome) and $66 \%$ were losing ('no goal') trials (i.e., the non-favorable outcome). The deterministic condition was designed so that a winning trial would occur on every third trial. In the probabilistic condition winning trials were programmed to occur on every third trial with a probability of 0.66 and on other trials with a probability of 0.17 . Finally, in the unpredictable condition there was a one in three chance of a goal on every trial (see Table 1). Because of this probabilistic set up anumber of actual winning and losing trials could differ slightly between participants. Consequently caution had to be taken that these differences do not drive differences between groups. In our case we did find that by chance additional positive trials were present in PD group in probabilistic condition, and this was taken into account in the analyses. The assignment of the participants to one of the three conditions was randomized.

Participants' difference scores (Optimal Score-Actual Score) were analysed, and compared to the difference scores that would be expected if participants utilized the worst possible strategy [(Optimal Final Score-Actual Final Score)/(Optimal Final ScoreWorst Strategy Final Score)].This was followed by the analysis of the number of times participants followed the optimal strategy (bet low, low, high in Deterministic and Probabilistic conditions, and bet low, low, low in the Unpredictable condition) across trials. In addition, as the striatum has been associated with the development of Gambler's Fallacy (i.e., a decision-making bias that results in a tendency to increase a bet after a loss or decrease a bet after a win) [49] participants' betting behaviour was analysed with regard to whether they displayed Gambler's Fallacy. This was done using two measures: Bet Decrease measure [(No. of bet decreases after win at 30 not in third position divided by no. of wins at 30 not in third position)+(No. of bet decreases after win at 20 not in third position divided by no. of wins at 20 not in third position)]; and Bet Increase measure [(No. of bet increases after loss at 10 not in second position 
divided by no. of losses at 10 not in second position)+(No. of bet increases after loss at 20 not in second position divided by no. of losses at 20 not in second position)]. These measures take into account the types of strategies implemented in the Deterministic and Probabilistic conditions. They also take into account that gambler's fallacy cannot occur following a loss at 30 (only a stay or decrease is possible on the next trial, so there is no opportunity for an 'increase after a loss'), or following a win at 10 (only a stay or increase is possible on the next trial, so there is no opportunity for 'decrease after a win'). Finally, as part of analysing participant's betting behaviour, we also analysed the frequency of specific bets (i.e., how often a bet of 10,20 or 30 was made) on favorable and unfavorable trials separately.

The task was run on a 15" Sony Laptop. The experiment received ethical approval from the Joint Ethics Committee of the Institute of Neurology and The National Hospital for Neurology and Neurosurgery. Informed consent was obtained from all participants.

\section{Results}

Independent samples t-tests were used to compare demographic characteristics of the PD and OHC groups (Age, IQ, BDIII, MMSE). Since the number of males and females in each experimental group (PD, OHC, YHC) differed, performance of males and females on all dependent measures was also compared. No significant differences were found. Significant differences between PD patients and OHCs were found, however, in the results of BDI-II measure of depression $(\mathrm{p}=0.01)$ and MMSE measure of global cognitive functioning ( $\mathrm{p}=0.032$ ). Such differences are commonly found in studies comparing PD patients and OHCs $[\mathbf{2 2}, \mathbf{4 2}, \mathbf{5 0}-\mathbf{5 3}]$. As far as the BDI-II is concerned, excluding participants who scored above the cut-off score on this measure $(>18)$ did not alter the pattern of results obtained, so we decided to include these participants in our analysis. With regard to MMSE results, OHCs scored 29.62 (out of 30) on average on this test, whereas PD patients' average score was 29.04. This difference, even though significant, is not meaningful in terms of cognitive functioning of participants this is because the generally accepted MMSE cut off score is 24 [54] and both groups scored above this.

Nevertheless, to establish if the difference between participants on measures of depression and cognitive functioning had a bearing on participants' performance, an additional comparison of PD patients and OHCs results was performed with BDI-II and MMSE scores entered as covariates. No major change to the results was observed in response. The results of this additional analysis are presented in section 3.2. We also conducted a correlation analysis which revealed no relationship between the scores on the BDI-II questionnaire, scores on the MMSE test, and any of the dependent variables (Difference Score: BDI-II $\mathrm{p}=.0146$, MMSE $\mathrm{p}=0.451$; Worst Difference Score: BDI-II $\mathrm{p}=0.553$, MMSE $p=0.801$; Strategy: BDI-II $p=0.106$, MMSE $p=0.261$; Bet Decrease: BDI-II $p=0.131$, MMSE $p=0.85$; Bet Increase: BDI-II $\mathrm{p}=0.998$, MMSE $\mathrm{p}=0.23$ ).

\section{Comparison of YHCs, OHCs and PD patients Difference between optimal and final scores}

Since the predictability of the "winning trials" differed between conditions, which could lead to differences in the final scores achieved, to measure participants' general performance on the task the difference between final scores and optimal scores (i.e., scores that could be expected if participants used an optimal strategy throughout the task) was calculated. To examine the influence of the task condition and neurological impairment on the general performance of participants, a $3 \times 3$ ANOVA was conducted on these difference scores with Condition (Deterministic, Probabilistic, Unpredictable) and Group (PD patients, OHCs, YHCs) as independent variables. The analysis revealed no main effects of Group $\left(\mathrm{F}_{(2,86)}=1.07, \mathrm{p}=0.347\right)$ or Group $x$ Condition interaction $\left(\mathrm{F}_{(4,86)}=1.27, \mathrm{p}=0.287\right)$. There was, however, a significant main effect of Condition $\left(\mathrm{F}_{(2,86)}=35.16\right.$, $\mathrm{p}<0.001$ ), as indicated by Figure 1. Post-hoc analysis revealed significantly smaller deviations from the optimal score in the Unpredictable condition, as compared with the Deterministic $(\mathrm{p}<0.001)$ and Probabilistic conditions $(\mathrm{p}<0.001)$. This indicates that participants in the Unpredictable condition scored closer to the optimal score than participants in the other two conditions. Further analysis also revealed a trend for OHCs generally scoring further away from the optimal score than PD patients in the probabilistic condition $(\mathrm{M}=484, \mathrm{SD}=231.7$ vs. $\mathrm{M}=284.4$, $\mathrm{SD}=150.9 ; \mathrm{p}=0.055)$, but this was associated with a coincidental larger number of winning trials in PD group in this condition, and does not reflect genuine differences between groups in terms of how well they adapt to probabilistic environments.

\section{Comparison of the actual difference score to the difference score that would be expected if participants utilized the worst possible strategy}

When analysing the participants' difference scores in relation

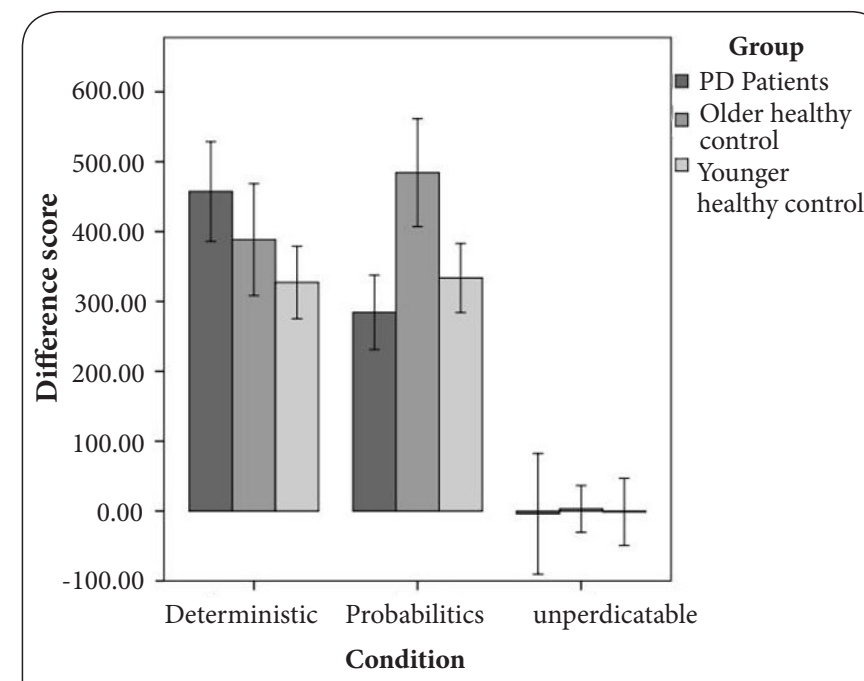

Figure 1. Difference between final scores and optimal scores in deterministic, probabilistic, and unpredictable conditions. 
to the worst possible difference scores in the three different conditions we found no significant differences between groups and conditions, and no interactions ( $p>0.05$ ), as suggested by Figure 2.

\section{Frequency of application of the optimal strategy}

To explore the extent to which optimal betting strategies were employed consistently throughout the 50 trials, we examined the number of times in which the optimal triplet was applied across conditions. In deterministic and probabilistic conditions the optimal triplet was defined as two bets that were less than

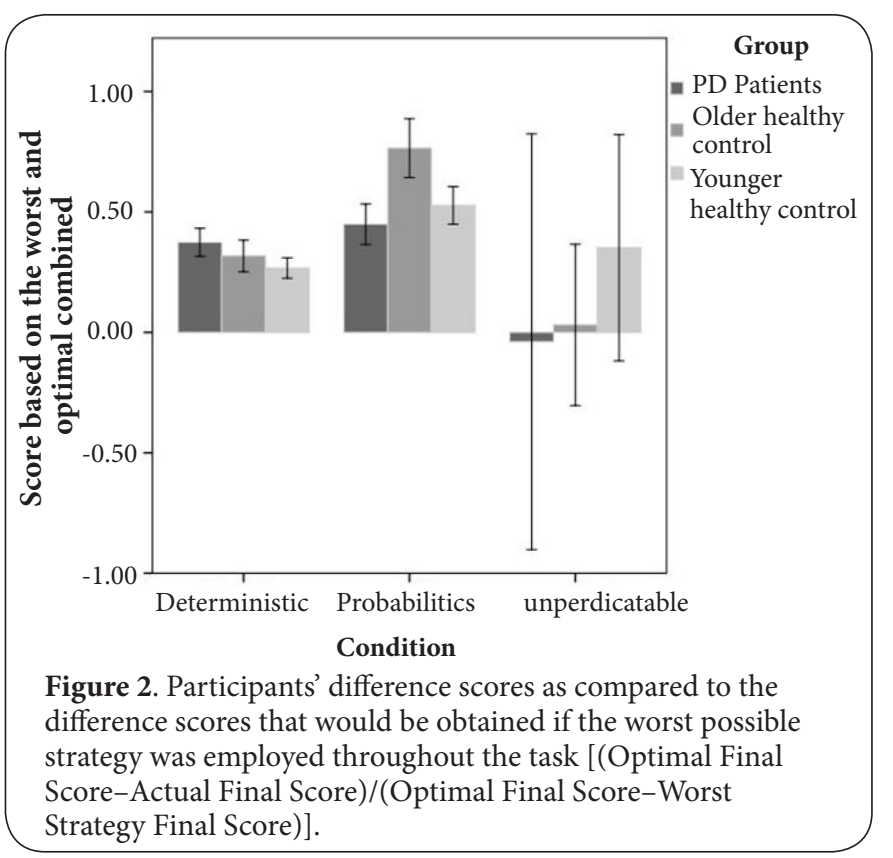

30 on non-favorable trials, and a bet of 30 on the favorable trial (e.g., 10, 10, 30, or 20, 20,30). In the unpredictable condition, the optimal triplet was defined as three consecutive bets of 10 . Because the analysis was based on triplets, we excluded the last two bets of the experiment -consequently a total of 48 trials were analysed. A 3x3 ANOVA using Condition and Group as factors showed that frequency with which the optimal betting strategy was employed in the task differed by Condition only $\left(\mathrm{F}_{(2,86)}=19.433\right.$, $\mathrm{p}<0.001)$ and that there was no main effect of Group. Post-hoc analysis revealed participants employed the optimal strategy significantly more often in the Deterministic condition than in the Probabilistic and Unpredictable conditions $(\mathrm{p}<0.001)$, as indicated by Figure 3. The number of 'triples' utilizing the optimal strategy in the Probabilistic and Unpredictable conditions was low $(\mathrm{M}=2.5, \mathrm{SD}=2.11$ and $\mathrm{M}=3.06, \mathrm{SD}=3.74$ out of 16 possible, respectively), suggesting that the optimal strategy was not implemented often. No other main effects were observed.

\section{Analysis of gambler's fallacy}

The results of a 3x3 ANOVA on Gambler's Fallacy measure of

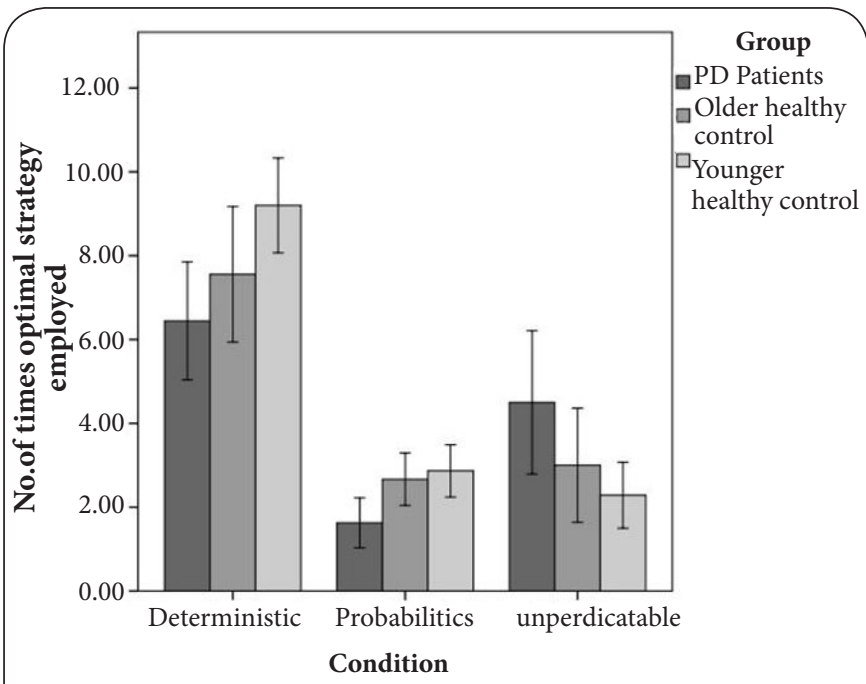

Figure 3. Strategy acquisition: the number of times participants followed the optimal strategy (bet low, low, high in the deterministic and random conditions, and bet low, low, low) throughout the task.

Bet Decreases were not significant. In contrast, a 3x3 ANOVA on Bet Increases revealed a significant effect of Condition $\left(\mathrm{F}_{(2,86)}=7.94, \mathrm{p}=0.001\right)$ and a trend towards Group $\mathrm{x}$ Condition interaction $\left(\mathrm{F}_{(4,86)}=2.027, \mathrm{p}=0.098\right)$. Post-hoc analysis showed that participants in the Probabilistic condition were more likely to increase their bet after a loss as compared to participants in the Deterministic $(\mathrm{p}=0.003)$ and Unpredictable conditions $(p=0.003)$. The analyses also revealed that in the Probabilistic condition there was a significant difference $(p<0.01)$ between PD patients and YHCs based on the number of bet increases after a loss, with PD patients more prone to increasing their bets after a loss than YHCs. Another aspect of betting in which PDs appeared to show more risk seeking behavior was the frequency of low bets on non-favorable trials. For each participant the frequency of low medium and high bets was analysed, which revealed that $\mathrm{PD}$ patients made low and medium bets less often on non-favorable trials than OHCs $(\mathrm{p}<0.001)$.

\section{Comparison of PD patients and OHCs, controlling for group differences in depression and global cognition scores}

Patients and OHCs were found to differ in terms of their scores on the BDI-II scale and the MMSE test. Therefore, additional comparisons of PD patients and OHCs were conducted which incorporated BDI-II and MMSE scores as covariates. The results of these analyses were in line with the results presented thus far.

\section{Difference between optimal and actual final scores (when MMSE and BDI-II scores entered as covariates)}

The analysis of difference scores with MMSE and BDI-II scores entered as covariates revealed no interaction between Group and Condition. There was, however, a significant main effect 
of Condition $\left(\mathrm{F}_{(2,43)}=25.16, \mathrm{p}<0.001\right)$ and Group $\left(\mathrm{F}_{(1,43)}=4.73\right.$, $\mathrm{p}=0.035$ ). Participants in the Unpredictable condition were found to score significantly closer to the optimal score than participants in both Deterministic and Probabilistic conditions (both $\mathrm{p}<0.001$ ). Post-hoc analyses also revealed that PD patients scored significantly closer $(\mathrm{p}=0.035)$ to the optimal score than OHCs $(M=200.4, S D=41.8$ vs. $M=337.5, S D=41.1)$. This was driven by the differences between groups in the Probabilistic condition ( $\mathrm{p}=0.02$ ), which suggests that this result might be due to a coincidental larger number of winning trials in PD group in this condition. No other significant effects were observed.

\section{Comparison of the actual difference score to the difference score that would be expected if participants utilized the worst possible strategy (when MMSE and BDI-II scores entered as covariates)}

In accordance with the pattern of results presented earlier, an analysis taking into account the participants' difference score in relation to the worst difference score possible revealed no differences between groups and conditions ( $p>0.05)$.

\section{Frequency of application of the optimal strategy} (when MMSE and BDI-II scores entered as covariates) A $3 \times 3$ ANOVA on Strategy, using Condition and Group as factors showed that frequency with which the optimal betting strategy was employed in the task differed by condition only $\left(\mathrm{F}_{(2,43)}=11.267, \mathrm{p}<0.001\right)$ and that there was no main effect of Group, which is consistent with the results of the main analysis. Participants in the deterministic condition utilized the optimal strategy significantly more often than those in the Probabilistic $(\mathrm{p}<0.001)$ and Unpredictable $(\mathrm{p}=0.017)$ conditions. There was no significant difference in the number of times the optimal strategy was used between Probabilistic and Unpredictable condition $(\mathrm{p}=0.854)$.

\section{Analysis of Gambler's fallacy (when MMSE and BDI-II scores entered as covariates)}

Analysis of Gambler's Fallacy measures revealed no significant effect of Condition or Group on Bet Decreases. It has, however, revealed a significant main effect of Condition on Bet Increases $\left(\mathrm{F}_{(2,43)}=6.114, \mathrm{p}=0.005\right)$. Further analysis revealed that participants in the Probabilistic condition were more likely to increase their bet after a loss than participants in the Deterministic and Unpredictable conditions ( $\mathrm{p}=0.024$ and $\mathrm{P}=0.006$, respectively). As in previous analyses, PD patients were found to be less likely than OHCs to bet 10 in the Probabilistic condition, and less likely to bet 10 or 20 in all three conditions, indicating that they were more risk seeking than the other two groups.

\section{Discussion}

The aim of this study was twofold. The first main aim was to establish how PD patients perform in comparison to two types of control groups in probabilistic, deterministic and unpredictable versions of the same task. The second aim was to examine in detail patterns in performance when taking into account metrics that focus on what is optimal and sub-optimal in a deterministic, probabilistic and unpredictable condition.

\section{Pattern of findings between groups}

Across several different methods of analysis of performance, the results of the study suggest that $\mathrm{PD}$ patients are able to perform just as well as OHCs when the rule governing the cue-outcome relationship is deterministic or unpredictable. The study also shows that PD patients can perform just as well as HCs (both young and age-matched) when the cue-outcome relationship is probabilistic. This suggests that probabilistic task set ups per se are not the critical factor in inducing impaired decision-making in patients with PD. Nevertheless, this requires some explanation.

In our study people with PD were more likely than the other groups to behave in a risky manner; this was indicated by their tendency to increase their bet size after losses (i.e., Gambler's Fallacy). In addition, PD patients tended to stick to higher bets consistently across trials. This suggests that PD patients were more risk-seeking than OHCs in our experiment, which is in line with the results obtained from IGT tasks. Studies using the IGT task show that when compared to OHCs, PD patients are more likely to choose an option associated with high reward seven though they also have higher losses attached to them $[9,50,51,55]$.

This result sheds some light on the nature of decisionmaking deficits of PD patients in probabilistic conditions. It is not the case that PD patients are simply sub-optimal in their decision-making with respect to this condition, instead, they show nuanced behavior with respect to the actual cue-outcome association. However, as PD progresses, changes occur in cognitive functioning (e.g., increased perseverance or decreased sensitivity to negative feedback), which in turn predisposes $\mathrm{PD}$ patients to adopting certain strategies (e.g., those that involve greater risk-taking) more often than OHCs [10]. This has consequences for patients' performance on different decisionmaking tasks. For example PD patients have repeatedly been shown to perform suboptimally compared to OHCs on a standard IGT task which requires participants to avoid options associated with high but risky wins $[\mathbf{4 , 6 , 5 6}]$. However, when the structure of the IGT is altered, so that the more risky option becomes the more advantageous one, PD patients are thought to perform just as well as OHCs [55]. This shows that the tendency to adopt risky strategies that develops in Parkinson's disease can actually be beneficial in certain circumstances. It also shows that how well PD patients do on a decision-making task depends, at least to some extent, on the strategy leading to optimal performance within this task. If the optimal strategy coincides with the pattern of behaviour PD patients are likely to exhibit due to changes in dopamine levels in the brain, then performance will be intact. If it goes against this pattern, performance will be likely to be impaired. This highlights the importance of 
defining what an optimal performance actually is within a task when investigating decision-making in PD. Furthermore, our results point to the importance of taking into account the costs of utilizing the suboptimal strategy throughout the task. On our task risky betting was not an optimal strategy, but the costs of utilizing this strategy were relatively low. We speculate that in the present study PD patients' performance was as good as that of OHCs because utilizing a suboptimal strategy in this particular environment was not particularly costly. This means that how well PD patients do on a decision-making task not only depends on the optimal strategy within the task, but also how costly it is not to utilize this strategy.

In sum, our findings suggest that probabilistic cue-outcome relationship is not the critical factor in causing decision-making deficits in PD. What matters as a basis for explaining deficits in decision-making performance in PD is the cost of not utilising an optimal strategy. In probabilistic task setups in which the cost is high (e.g., IGT), performance appears to be poor. In task setups in which costs are low (e.g., unpredictable condition in the present study), performance seems to be unaffected. This means that the decision-making environment, particularly the cost associated with utilising suboptimal strategies, plays an important role. In addition, this interacts with changes in cognitive functioning in PD that affect the choice of strategy in decision-making tasks. If the optimal decision-making strategy is in line with the cognitive changes occurring in PD (e.g., the optimal strategy requires making risky choices, and PD patients are inclined to be more risk-seeking), performance will be unaffected. If, however, the optimal decision making strategy is incongruent with the changes occurring in PD (e.g., the optimal strategy requires avoiding risky choices, and PD patients are inclined to be more risk-seeking) performance will be compromised.

\section{General pattern of findings across groups}

The results of this experiment provide a strong case for establishing what should constitute optimal behavior in an environment. As expected, considering the small costs of employing the 'worst strategy' in the unpredictable condition, there was sufficient flexibility for participants to learn with minimal cost in making errors, and so scores were close to optimal. In the deterministic condition, on the other hand, the high cost of employing erroneous strategies meant that most participants fell far from the optimal score. In the probabilistic condition most participants (both PD patients and YHC and OHC groups) also failed to apply the optimal strategy, which was reflected in the large deviation from the optimal score.

If we turn our attention to strategy discovery, what was evident from our analyses is that there were no differences between the probabilistic and unpredictable conditions. However, that is not to say that participants treated the probabilistic condition as if it were unpredictable. Further analysis of the betting strategies revealed that participants in the probabilistic condition were much more likely to increase their bets after a loss than participants in the unpredictable condition. This suggests that, overall across all three groups, people are sensitive to probabilistic cue-outcome associations, to the extent that they do not behave as if they were random, but they find it difficult to uptake of the optimal strategy. Again, it is worth highlighting that the relative difference in cost of error between the unpredictable and probabilistic condition will also impact on the acquisition of and application of strategies and will consequently affect performance in different ways; which was revealed in this study.

\section{Conclusions}

In the present study PD patients' performance was compared to young and older HCs in three different versions of the same gambling task: deterministic, probabilistic, and unpredictable. In all three groups betting behavior was assessed according to optimality. The results of the study show that, overall, PD patients perform as well as OHCs and YHCs. We show that two important factors should be taken into account when interpreting $\mathrm{PD}$ patients' performance:1) the relative cost of error and success, and 2) PD patients' choice behavior as a function of cognitive changes that predispose them adopting different strategies. Taking them into account provides an explanation for why PD patients show impaired performance on some probabilistic tasks, such as WPT or IGT, but also good performance on other tasks, such as the gambling task used in this study. Our results point to the importance of defining what good performance actually means within a task, not only through utilising reference groups such as OHCs and YHCs, but also through establishing an optimal performance metric for that task.

\section{Competing interests}

The authors declare that they have no competing interests.

\section{Authors' contributions}

All authors contributed equally to the manuscript.

\section{Publication history}

Editor:Vinay Parikh, Temple University, USA.

Received: 25 July 2014 Revised: 02 October 2014

Accepted: 20 October 2014 Published: 28 October 2014

\section{References}

1. Dirnberger $\mathrm{G}$ and Jahanshahi M. Executive dysfunction in Parkinson's disease: a review. J Neuropsychol. 2013; 7:193-224. I Article I PubMed

2. Marinus J, Visser M, Verwey NA, Verhey FR, Middelkoop HA, Stiggelbout $\mathrm{AM}$ and van Hilten JJ. Assessment of cognition in Parkinson's disease. Neurology. 2003; 61:1222-8. I Article I PubMed

3. McKinlay A, Grace RC, Dalrymple-Alford JC and Roger D. Characteristics of executive function impairment in Parkinson's disease patients without dementia. J Int Neuropsychol Soc. 2010; 16:268-77. | Article | PubMed

4. Czernecki V, Pillon B, Houeto JL, Pochon JB, Levy R and Dubois B. Motivation, reward, and Parkinson's disease: influence of dopatherapy. Neuropsychologia. 2002; 40:2257-67. | Article | PubMed

5. Gescheidt T, Czekoova K, Urbanek T, Marecek R, Mikl M, Kubikova R, Telecka S, Andrlova H, Husarova I and Bares M. lowa Gambling Task in patients with early-onset Parkinson's disease: strategy analysis. Neurol 


\section{Sci. 2012; 33:1329-35. | Article | PubMed}

6. Ibarretxe-Bilbao N, Junque C, Tolosa E, Marti MJ, Valldeoriola F, Bargallo $\mathrm{N}$ and Zarei $\mathrm{M}$. Neuroanatomical correlates of impaired decision-making and facial emotion recognition in early Parkinson's disease. Eur $J$ Neurosci. 2009; 30:1162-71. | Article | PubMed

7. Jahanshahi M, Wilkinson L, Gahir H, Dharmaindra A and Lagnado DA. Medication impairs probabilistic classification learning in Parkinson's disease. Neuropsychologia. 2010; 48:1096-103. | Article | PubMed

8. Knowlton BJ, Mangels JA and Squire LR. A neostriatal habit learning system in humans. Science. 1996; 273:1399-402. | Article | PubMed

9. Pagonabarraga J, Garcia-Sanchez C, Llebaria G, Pascual-Sedano B, Gironell $A$ and Kulisevsky J. Controlled study of decision-making and cognitive impairment in Parkinson's disease. Mov Disord. 2007; 22:1430-5. | Article | PubMed

10. Shohamy D, Myers CE, Onlaor S and Gluck MA. Role of the basal ganglia in category learning: how do patients with Parkinson's disease learn? Behav Neurosci. 2004; 118:676-86. | Article I PubMed

11. Ryterska A, Jahanshahi $M$ and Osman $M$. What are people with Parkinson's disease really impaired on when it comes to making decisions? A meta-analysis of the evidence. Neurosci Biobehav Rev. 2013; 37:2836-46. | Article | PubMed

12. Wilkinson L, Lagnado DA, Quallo $M$ and Jahanshahi M. The effect of feedback on non-motor probabilistic classification learning in Parkinson's disease. Neuropsychologia. 2008; 46:2683-95. | Article | PubMed

13. Sage JR, Anagnostaras SG, Mitchell S, Bronstein JM, De Salles A, Masterman D and Knowlton BJ. Analysis of probabilistic classification learning in patients with Parkinson's disease before and after pallidotomy surgery. Learn Mem. 2003; 10:226-36. | Article | PubMed Abstract | PubMed FullText

14. Perretta JG, Pari G and Beninger RJ. Effects of Parkinson disease on two putative nondeclarative learning tasks: probabilistic classification and gambling. Cogn Behav Neurol. 2005; 18:185-92. | Article | PubMed

15. Mattox ST, Valle-Inclan F and Hackley SA. Psychophysiological evidence for impaired reward anticipation in Parkinson's disease. Clin Neurophysiol. 2006; 117:2144-53. | Article | PubMed

16. Moody TD, Bookheimer SY, Vanek Z and Knowlton BJ. An implicit learning task activates medial temporal lobe in patients with Parkinson's disease. Behav Neurosci. 2004; 118:438-42. | Article | PubMed

17. Bechara A, Damasio AR, Damasio $H$ and Anderson SW. Insensitivity to future consequences following damage to human prefrontal cortex. Cognition. 1994; 50:7-15. | Article | PubMed

18. Knowlton BJ, Squire LR and Gluck MA. Probabilistic classification learning in amnesia. Learn Mem. 1994; 1:106-20. | PubMed

19. Shohamy D, Myers CE, Kalanithi J and Gluck MA. Basal ganglia and dopamine contributions to probabilistic category learning. Neurosci Biobehav Rev. 2008; 32:219-36. | Article | PubMed Abstract | PubMed FullText

20. Witt K, Nuhsman A and Deuschl G. Dissociation of habit-learning in Parkinson's and cerebellar disease. J Cogn Neurosci. 2002; 14:493-9. Article | PubMed

21. Brand M, Labudda K, Kalbe E, Hilker R, Emmans D, Fuchs G, Kessler J and Markowitsch HJ. Decision-making impairments in patients with Parkinson's disease. Behav Neurol. 2004; 15:77-85. | Article | PubMed Abstract | PubMed FullText

22. Euteneuer F, Schaefer F, Stuermer R, Boucsein W, Timmermann L, Barbe MT, Ebersbach G, Otto J, Kessler J and Kalbe E. Dissociation of decisionmaking under ambiguity and decision-making under risk in patients with Parkinson's disease: a neuropsychological and psychophysiological study. Neuropsychologia. 2009; 47:2882-90. | Article | PubMed

23. Gescheidt T, Marecek R, Mikl M, Czekoova K, Urbanek T, Vanicek J, Shaw DJ and Bares M. Functional anatomy of outcome evaluation during lowa Gambling Task performance in patients with Parkinson's disease: an fMRI study. Neurol Sci. 2013; 34:2159-66. | Article | PubMed

24. Thiel A, Hilker R, Kessler J, Habedank B, Herholz K and Heiss WD. Activation of basal ganglia loops in idiopathic Parkinson's disease: a PET study. J Neural Transm (Vienna). 2003; 110:1289-301. | Article | PubMed

25. Castrioto A, Funkiewiez A, Debu B, Cools R, Lhommee E, Ardouin C, Fraix $V$, Chabardes S, Robbins TW, Pollak P and Krack P. lowa gambling task impairment in Parkinson's disease can be normalised by reduction of dopaminergic medication after subthalamic stimulation. J Neurol Neurosurg Psychiatry. 2015; 86:186-90. | Article | PubMed

26. Cools R, Barker RA, Sahakian BJ and Robbins TW. Enhanced or impaired cognitive function in Parkinson's disease as a function of dopaminergic medication and task demands. Cereb Cortex. 2001; 11:1136-43. | PubMed

27. Cools R, Stefanova E, Barker RA, Robbins TW and Owen AM Dopaminergic modulation of high-level cognition in Parkinson's disease: the role of the prefrontal cortex revealed by PET. Brain. 2002; 125:58494. | PubMed

28. Cools R, Barker RA, Sahakian BJ and Robbins TW. L-Dopa medication remediates cognitive inflexibility, but increases impulsivity in patients with Parkinson's disease. Neuropsychologia. 2003; 41:1431-41. | Article I PubMed

29. Frank MJ, Samanta J, Moustafa AA and Sherman SJ. Hold your horses: impulsivity, deep brain stimulation, and medication in parkinsonism. Science. 2007; 318:1309-12. | Article | PubMed

30. Gotham AM, Brown RG and Marsden CD. 'Frontal' cognitive function in patients with Parkinson's disease 'on' and 'off' levodopa. Brain. 1988; 111 ( Pt 2):299-321. | PubMed

31. Cools R, Altamirano L and D'Esposito M. Reversal learning in Parkinson's disease depends on medication status and outcome valence. Neuropsychologia. 2006; 44:1663-73. | Article | PubMed

32. Frank MJ, Seeberger LC and O'Reilly R C. By carrot or by stick: cognitive reinforcement learning in parkinsonism. Science. 2004; 306:1940-3. | Article | PubMed

33. Kapogiannis D, Mooshagian E, Campion P, Grafman J, Zimmermann TJ, Ladt KC and Wassermann EM. Reward processing abnormalities in Parkinson's disease. Mov Disord. 2011; 26:1451-7. | Article | PubMed Abstract | PubMed FullText

34. Rutledge RB, Lazzaro SC, Lau B, Myers CE, Gluck MA and Glimcher $P W$. Dopaminergic drugs modulate learning rates and perseveration in Parkinson's patients in a dynamic foraging task. J Neurosci. 2009; 29:15104-14. | Article | PubMed Abstract | PubMed FullText

35. Shohamy D, Myers CE, Geghman KD, Sage J and Gluck MA. L-dopa impairs learning, but spares generalization, in Parkinson's disease. Neuropsychologia. 2006; 44:774-84. | Article | PubMed Abstract | PubMed FullText

36. Swainson R, Rogers RD, Sahakian BJ, Summers BA, Polkey CE and Robbins TW. Probabilistic learning and reversal deficits in patients with Parkinson's disease or frontal or temporal lobe lesions: possible adverse effects of dopaminergic medication. Neuropsychologia. 2000; 38:596-612. | Article | PubMed

37. Torta DM, Castelli L, Zibetti M, Lopiano L and Geminiani G. On the role of dopamine replacement therapy in decision-making, working memory, and reward in Parkinson's disease: does the therapy-dose matter? Brain Cogn. 2009; 71:84-91. | Article | PubMed

38. Osman M, Wilkinson L, Beigi M, Castaneda CS and Jahanshahi M. Patients with Parkinson's disease learn to control complex systems via procedural as well as non-procedural learning. Neuropsychologia. 2008; 46:2355-63. | Article | PubMed

39. Witt K, Daniels C, Daniel V, Schmitt-Eliassen J, Volkmann J and Deuschl G. Patients with Parkinson's disease learn to control complex systems-an indication for intact implicit cognitive skill learning. Neuropsychologia. 2006; 44:2445-51. | Article | PubMed

40. Osman M, Ryterska A, Karimi K, Tu L, Obeso I, Speekenbrink M and Jahanshahi M. The effects of dopaminergic medication on dynamic decision making in Parkinson's disease. Neuropsychologia. 2014; 53:15764. | Article | PubMed

41. Labudda K, Brand M, Mertens M, Ollech I, Markowitsch HJ and Woermann FG. Decision making under risk condition in patients with Parkinson's disease: a behavioural and fMRI study. Behav Neurol. 2010; 
Osman et al. Journal of Psychiatry and Brain Functions 2014,

http://www.hoajonline.com/journals/pdf/2055-3447-1-6.pdf

23:131-43. | Article | PubMed Abstract | PubMed FullText

42. Schmitt-Eliassen J, Ferstl R, Wiesner C, Deuschl G and Witt K. Feedbackbased versus observational classification learning in healthy aging and Parkinson's disease. Brain Res. 2007; 1142:178-88. | Article | PubMed

43. Shohamy D, Myers CE, Grossman S, Sage J, Gluck MA and Poldrack RA. Cortico-striatal contributions to feedback-based learning: converging data from neuroimaging and neuropsychology. Brain. 2004; 127:851-9. | Article | PubMed

44. Rudrauf D, Bennequin D, Granic I, Landini G, Friston K and Williford K. A mathematical model of embodied consciousness. J Theor Biol. 2017; 428:106-131. | Article | PubMed

45. Folstein MF, Folstein SE and McHugh PR. "Mini-mental state”. A practical method for grading the cognitive state of patients for the clinician. $J$ Psychiatr Res. 1975; 12:189-98. | Article | PubMed

46. Beck A.T, Steer R.A, Brown G.K. Manual for the Beck depression inventory-II, San Antonio, TX: Psychological Corporation. 1996.

47. Hoehn MM and Yahr MD. Parkinsonism: onset, progression and mortality. Neurology. 1967; 17:427-42. I PubMed

48. Schwab R.S, England A.C. Projection technique for evaluating surgery in Parkinson's disease. In: Gillingham, F.J., Donaldson, M.C., eds. Third symtposium on Parkinlson's disease. Edinburgh: Livingstone. 1969; 152157.

49. Xue G, Lu Z, Levin IP and Bechara A. An fMRI study of risk-taking following wins and losses: implications for the gambler's fallacy. Hum Brain Mapp. 2011; 32:271-81. | Article | PubMed Abstract | PubMed FullText

50. Kobayakawa M, Koyama S, Mimura M and Kawamura M. Decision making in Parkinson's disease: Analysis of behavioral and physiological patterns in the lowa gambling task. Mov Disord. 2008; 23:547-52. | Article | PubMed

51. Mimura M, Oeda R and Kawamura M. Impaired decision-making in Parkinson's disease. Parkinsonism Relat Disord. 2006; 12:169-75. | Article | PubMed

52. Poletti M, Frosini D, Lucetti C, Del Dotto P, Ceravolo R and Bonuccelli U. Decision making in de novo Parkinson's disease. Mov Disord. 2010; 25:1432-6. | Article | PubMed

53. Seo $\mathrm{H}$ and Lee $\mathrm{D}$. Temporal filtering of reward signals in the dorsal anterior cingulate cortex during a mixed-strategy game. J Neurosci. 2007; 27:8366-77. | Article | PubMed Abstract | PubMed FullText

54. Dick JP, Guiloff RJ, Stewart A, Blackstock J, Bielawska C, Paul EA and Marsden $C D$. Mini-mental state examination in neurological patients. J Neurol Neurosurg Psychiatry. 1984; 47:496-9. | Article | PubMed Abstract | PubMed FullText

55. Kobayakawa M, Tsuruya N and Kawamura M. Sensitivity to reward and punishment in Parkinson's disease: an analysis of behavioral patterns using a modified version of the lowa gambling task. Parkinsonism Relat Disord. 2010; 16:453-7. | Article | PubMed

56. Delazer M, Sinz H, Zamarian L, Stockner H, Seppi K, Wenning GK, Benke $T$ and Poewe W. Decision making under risk and under ambiguity in Parkinson's disease. Neuropsychologia. 2009; 47:1901-8. | Article | PubMed

\section{Citation:}

Ryterska A, Harris AJL, Ejova A, Jahanshahi M and Osman M. Investigating decision-making in Parkinson's disease in tasks characterized by probabilistic cueoutcome associations: the role of optimality. J Psychiatry Brain Funct. 2014; 1:6.

http://dx.doi.org/10.7243/2055-3447-1-6 